\title{
About Still Nonignorable Consequences of (Partially) Ignoring Missing Item Responses in Large-scale Assessment
}

\author{
Alexander Robitzsch ${ }^{1,2 *(D)}$ \\ 1 IPN - Leibniz Institute for Science and Mathematics Education, Kiel, Germany \\ 2 Centre for International Student Assessment (ZIB), Kiel, Germany \\ * Correspondence: robitzsch@leibniz-ipn.de
}

\begin{abstract}
In recent literature, alternative models for handling missing item responses in large-scale assessments are proposed. In principle, based on simulations and arguments based test theory (Rose 2013). In those approaches, it is argued that missing item responses should never be scored as incorrect, but rather treated as ignorable (e.g., Pohl et al. 2014). The present contribution shows that these arguments have limited validity and illustrates the consequences in a country comparison in the PIRLS 2011 study. A different treatment of missing item responses than recoding them as incorrect leads to significant changes in country rankings, which induces non-ignorable consequences regarding the results' validity. Additionally, two alternative item response models based on different assumptions for missing item responses are proposed.
\end{abstract}

Keywords: item response model, missing item responses, large-scale assessment, PIRLS

This article primarily relies on a previous book chapter in German language (Robitzsch 2016).

\section{Introduction}

In large-scale assessment studies such as PIRLS, TIMSS, or PISA, students' competencies are assessed using test items. Often, however, students do not respond to specific items (missing item responses), and it is unclear how item nonresponse should be treated in the computation of values of competencies (i.e., values of the latent trait).

While the treatment of missing data in statistical analyses in social sciences is now widely used (Graham 2009), in recent literature, there is criticism of conventional methods of treating missing item responses in item response models (IRT models) in large-scale assessment studies (Pohl et al. 2014; Rose et al. 2010). Typically, the treatment of item responses can be distinguished between the process of calibration (determination of item parameters) and scoring (determination of ability distributions).

In PIRLS, TIMSS, and PISA (until PISA 2012), only missing item responses at the end of a test booklet (so-called not reached items) are omitted (i.e., ignored) in calibration in order not to distort estimates of item parameters. In scoring, missing item responses are evaluated as incorrect. Since PISA 2015, not reached items are ignored in calibration and scoring (although the proportion of missing items is used as a predictor in the latent regression model).

However, researchers Steffi Pohl (Pohl and Carstensen 2012 2013; Pohl et al. 2014) and Norman Rose (Rose 2013) argued that missing item responses should never be treated as incorrect (i.e., in calibration as well as in scoring). These researchers proposed alternative item response models to handle missing item responses properly. The following article will first elaborate on the criticisms made in the literature (Pohl et al. 2014; Rose 2013) to treat missing item responses as incorrect and the proposed model-based alternatives. In contrast to the above literature, we argue that only for reasons of validity and not for test-theoretical reasons, a particular method for handling (i.e., both in calibration as well in scaling) should be preferred. Since assumptions about the missingness of item responses are not empirically testable, we propose two alternative item response models 
that directly parametrize different assumptions of the missingness process. Finally, we discuss potential psychometric consequences in large-scale assessment for assessing competencies with varying treatments of missing item responses.

\section{An Analysis of Points of Criticism of Traditional Approaches to Handling Missing Item Responses}

Typically, missing item responses are scored as incorrect for determining ability distributions in large-scale assessment studies. In a series of recent publications, it is claimed that missing item responses should never be scored as incorrect, and it is recommended that this traditional ad hoc method should never be used in large-scale assessment studies (Pohl and Carstensen 2012 2013; Pohl et al. 2014; Rose 2013). In the following, we discuss the authors' arguments and conclude that they are based on assumptions and conclusions that are atypical in the application of item response models. Therefore, we find the recommendations in the above-mentioned literature somewhat doubtful.

\subsection{Aleatoric and Epistemic Uncertainty}

Useful for the following considerations is the distinction of Denoeux (2011a) in the quantification of the uncertainty in data (the item responses) and corresponding models. He distinguishes the concepts of probability and possibility. The uncertainty due to sampling or the modeling of existing data (a dataset with all item responses) is denoted as aleatoric uncertainty. Uncertainty (or fuzziness) for every single date (i.e., each individual item response) is denoted as epistemic uncertainty. Denoeux (2011a) argues that aleatoric uncertainty can be expressed by probabilities in a statistical model. In the case of large-scale assessment studies, this means that an IRT model for the dataset of item responses with fixed values is fitted. Each student's individual item responses are viewed under the concept of epistemic uncertainty, which would result in fuzzy item responses (Denoeux 2011ab). Therefore, it is unclear how one should score missing item responses. Nevertheless, in this interpretation, it is evident that assumptions on the individual data points (i.e., the individual item responses) must be strictly distinguished from the assumptions in a probability model, which is a statistical model for item responses of all students. To illustrate this conceptual distinction, we assume that the distribution of the running times in a 50-meter run for a defined population of Austrian students in the 4th grade. For this purpose, a sample of students is drawn. The associated uncertainty of sampling students from the population is associated with probabilities in the context of aleatoric uncertainty. However, the measurement of the running time of a student can never be precisely carried out (e.g., due to measurement uncertainty, the runtime is located to be in the interval between 8.75 and 8.85 seconds, which represents a fuzzy date). This kind of uncertainty corresponds to the concept of epistemic uncertainty. To sum up, assumptions about the emergence of data (aleatoric uncertainty) must be distinguished from assumptions about the modeling of existing data (epistemic uncertainty). It does not seem wise to mix up these two processes. Unfortunately, this is precisely the case in the reasoning of why missing item responses should not be scored as incorrect (Pohl and Carstensen 2012 2013; Rose 2013; Rose et al. 2010).

\subsection{Reasoning Based on Test Theory}

The criticism of treating missing item responses as incorrect is based on simulations and test-theoretical arguments (Rose 2013), which we will discuss in detail below.

Most simulation studies convey that scoring of missing item responses as incorrect provides distorted item parameters and ability distributions (Rohwer 2013). In these simulation studies, a missing item response is mostly simulated due to a dependence on latent variables or person covariates, but not depending on the item itself. Then, the model parameter's claimed biasedness by treating missing item responses as incorrect obviously follows because the simulation is based on a data generating model that is not in correspondence with the analysis model (i.e., treating missing item responses as incorrect). In contrast, if a particular IRT model fits, and missing values are generated 
for all items, for which the item was solved incorrectly, then scoring missing item responses anything from incorrect will provide distorted model parameters (see Rohwer 2013). Whether simulation-based reasoning against the scoring of missing item response as incorrect is valid depends on the plausibility of the data generating model for the missingness process. We argue that approaches that model missingness on an item independently on the unknown item response itself are implausible. Thus, simulation studies are not helpful for the justification for choosing an adequate treatment of missing item responses in real data.

In test-theoretical reasoning, the core of the criticism is that scoring missing item responses as incorrect would model missing item responses deterministically. This modeling strategy would contradict with model implied item response probabilities in an IRT model, and it is therefore invalid (Pohl et al. 2014; Rose 2013). Let us denote the missingness indicator variable with $R_{p i}$, that for a person $p$ an item $i$ is observed (i.e., $R_{p i}=1$ ). Then, formally, for the incorrect scoring, a missing item response $Y_{p i}$ is set to zero, and, thus, $P\left(Y_{p i}=1 \mid R_{p i}=0\right)=0$ independent of the latent trait $\theta_{p}$ of person $p$. This would lead to a contradiction because the prediction in the IRT model (e.g., in a 2PL model) would result in a probability $P\left(Y_{p i}=1 \mid \theta_{p}\right)>0$, i.e., the data handling is deterministic, but the IRT model is probabilistic (Rose 2013). Hence, model assumptions in the IRT model would be violated. The contradiction can be tackled by using the different concepts of uncertainty, according to Denoeux (2011a), because in the argumentation of Rose (2013) and Pohl et al. (2014), epistemic and aleatory uncertainty are confounded. In this literature, the same probability model (i.e., the item response probability from an IRT model) for the occurrence of a single data point and modeling all item responses in the sample is employed.

If we embed our considerations now more firmly in concepts in psychometrics, it is noticeable that the rejection for treating missing item responses as incorrect relies on an intraindividual interpretation of the probabilities in the IRT model. This reasoning corresponds to a stochastic subject perspective (i.e., probabilities of the IRT model can be interpreted for each combination of a person $p$ and an item $i$; see Holland 1990), which is not typically employed in large-scale assessment studies (Wainer 2010). If one uses a random sampling perspective (Holland 1990; Molenaar 1995), the probabilities are interpreted as the result of a sampling of persons, and item parameters as well as a latent trait distribution for $\theta$ are introduced as a representation of a high-dimensional contingency table for discrete multivariate observations $Y$. Then, formally, the IRT model can be written as

$$
P\left(\boldsymbol{Y}=\left(y_{1}, \ldots, y_{I}\right)\right)=\int \prod_{i=1}^{I} P\left(Y_{i}=y_{i} \mid \theta\right) f(\theta) \mathrm{d} \theta
$$

In this notation, it becomes clear that in IRT models, a distribution is modeled for a sample of persons, and not individuals are represented (see also Rohwer 2013). If, however, one assumes the intraindividual perspective in the sense of the stochastic subject perspective, the reasoning is based on the assertion that the scoring of missing item responses as incorrect would lead to a deterministic item response probability of zero because the scoring as incorrect leads to a wrong item response with a probability of one (i.e., $\left.P\left(Y_{p i}=0 \mid R_{p i}=0, \theta_{p}\right)=1\right)$. However, the scoring of an item response as incorrect is not related to the probabilistic modeling of item responses of a person. This is because the missing item responses could also have been generated by a deterministic process, which does not contradict the probabilistic modeling in the statistical model for all item responses of all persons. Therefore, one has to distinguish between a real response process - which can be either deterministic or probabilistic but certainly follows the given probabilistic specification of the IRT model in only rare cases - and the statistical model assumption for the definition of ability in terms of a scale construction (Rohwer 2013).

Also, statistical reasons from the stochastic subject perspective invalidate the criticism of scoring missing item responses as incorrect. When modeling intraindividual distributions in an IRT model for a person, item parameters are defined as fixed and known parameters, and the latent trait $\theta_{p}$ is required as a fixed effect that is assigned to a person. Then, the assumption of local stochastic Independence 
refers to conditional independence of item responses for a fixed person $p$ based on a set of items, and it is therefore not empirically falsifiable, but a means for identifying the ability $\theta_{p}$ of this person. Hence, the ability $\theta_{p}$ is only defined by the specification of likelihood and item responses $Y$. In the Rasch model, for a fixed person $p$ and item $i$, the probability of a correct item response is given by $P\left(Y_{p i}=1 \mid \theta_{p}\right)=\Psi\left(\theta_{p}-b_{i}\right)$ (where $\Psi$ denotes the logistic function), which is used in the likelihood estimation for the distribution of the trait $\theta_{p}$. Whether a single observation then "fits" in this model is empirically undecidable, so that scoring missing item responses as incorrect does not necessarily violate the IRT model.

To sum up, in our opinion, the reasonings given in the literature (Pohl et al. 2014; Rose 2013) for not scoring missing item responses as incorrect that are based on simulation studies or test theory rationales does not seem to be typical for applications in large-scale assessment studies. However, we note that for reasons of validity, quite different scoring methods than as incorrect could be adequate in applications. Central in the argumentation of the authors' criticism is that modeling the response processes of students in a cross-sectional measurement (interindividual perspective) is erroneously confounded with response processes associated with a single person (intraindividual perspective; see Molenaar 2004).

\section{Model Based Treatment of Missing Item Responses}

In this section, different model based approaches for the treatment of missing item responses are discussed. In the following, we only consider IRT models for dichotomous data in Rasch models (Fischer and Molenaar 1995). However, our considerations are also applicable to more general model classes such as 2PL models or IRT models for polytomous data (Yen and Fitzpatrick 2006).

Among the model based procedures, IRT models are often used for distinguishing between ignorable and nonignorable item responses (Holman and Glas 2005). By ignoring missing item responses items for students in the likelihood function, missing item responses are omitted in the estimation. If one ignores missing item responses in the estimation, it can be shown that the missing item responses can be imputed (i.e., scored) under this assumption with a probability of $P_{p i}\left(\theta_{p, M}\right)$ (i.e., scored) as correct. Here, $\theta_{p, M}$ denotes the ability of person $p$ that is being calculated exclusively with the observed item responses. This practically means that in the IRT model, observed item responses can be used to impute missing item responses. Since the probability $P_{p i}\left(\theta_{p, M}\right)$ is always larger than zero, ignoring missing item responses always leads to larger estimated trait values $\theta_{p}$ than scoring them as incorrect. Ignoring missing item responses does not mean that the item nonresponse is independent of the skill (i.e., the missingness is not missing completely at random, MCAR; see Graham 2009). The missing process is characterized by the fact that the whole information about the ability $\theta_{p}$ is already recoverable from the observed item responses, i.e., the missing data are missing at random (MAR). Under this assumption, the proportion of missing item responses might be larger for students with lower abilities than for students with higher abilities.

The treatment of missing item responses as incorrect can be considered one extremum, ignoring them as the alternative pole. In the first case, there is a risk of underestimating the ability to be a person. In the second case, there is a risk of overestimating the ability to be a person.

As an alternative to ignoring missing item responses, multidimensional IRT models for nonignorable item responses have been proposed (Debeer et al. 2017; Frey et al. 2018; Fu et al. 2010; Glas et al. 2015; Holman and Glas 2005; Köhler et al. 2015a; Kreitchmann et al. 2018; Kuha et al. 2018; Lu and Wang 2020; Okumura 2014; Pohl and Becker 2020; Rosas and Shomer 2008; Rose et al. 20152017 2010; Santos et al. 2016; Ulitzsch et al. 2020; Weeks et al. 2016; Zhou and Huggins-Manley 2019). The two-dimensional model of Holman and Glas (2005) introduces a latent individual response propensity (response tendency) $\xi$ (besides the latent ability $\theta$ ), which predicts missingness in item responses. For the response variables $R_{p i}$, a Rasch model is assumed

$$
P\left(R_{p i}=1 \mid \theta_{p}, \xi_{p}\right)=\Psi\left(\xi_{p}-\beta_{i}\right)
$$


The probability of responding to an item, therefore, depends on the response tendency $\xi_{p}$ and from a parameter $\beta_{i}$. Whether an item is missing or but does not depend on the unknown item response itself. This means that $Y_{p i}$ and $R_{p i}$ are conditionally independent given $\theta_{p}$ and $\xi_{p}$. The complete information for model parameters can be extracted from observed item responses and response indicators by specifying a particular parametric model. Formally, missing data are missing not at random (MNAR). However, missingness can be traced back to a further latent variable. This kind of missingness process is also denoted as latent ignorability (Harel and Schafer 2009; see also Bartolucci et al. 2018, Beesley et al. 2019, Jung et al. 2011).

In the two-dimensional model, a bivariate distribution for $(\theta, \xi)$ is estimated (Holman and Glas 2005; Köhler et al. 2015b; Rose et al. 2010). Alternative model based procedures generalize the two-dimensional IRT model of Holman and Glas (see Rose 2013, Bertoli-Barsotti and Punzo 2013) or use mixture distribution approaches (Bacci and Bartolucci 2015; Bacci et al. 2017). It should be emphasized that for an individual person, the maximum likelihood estimate for the ability $\theta_{p}$ does not exist, and can only use the EAP estimate (Yen and Fitzpatrick 2006). As a consequence, a correlation of the ability $\theta$ and the response tendency $\xi$ has to be known for estimating individual ability values.

If the response tendency $\xi_{p}$ can be expressed as $\xi_{p}=\rho \theta_{p}+\varepsilon_{p}$, one can show that missing item responses are approximately scored as $P_{p i}\left(\theta_{p, M}\right)-\rho$ (see Bertoli-Barsotti and Punzo 2013). In contrast to the model with ignorable item responses, the ability estimates are therefore adjusted by a constant $\rho$. Only if the response tendency and the ability are uncorrelated $(\rho=0)$, both models lead to the same ability estimates.

\section{Two Alternative Item Response Models for Nonignorable Item Responses: Approaches for a Sensitivity Analysis}

In this section, two alternative model-based approaches for nonignorable missing item responses are discussed. In the first model, missing item responses are scored as partially correct (Lord 1974). In the second IRT model, the two-dimensional IRT model of Holman and Glas (2005) is extended for the potential dependence of the missingness of an item from the unknown item response itself. In this model, the treatment of missing item responses as incorrect turns out to be a particular case. The two proposed models can be seen as a sensitivity analysis in which different assumptions about the missingness process can be investigated (e.g., Resseguier et al. 2011).

\subsection{Pseudo Likelihood Approach for Partially Correct Scoring of Missing Item Responses}

In the pseudo likelihood approach of Lord (1974), missing item responses are scored as partially correct. The pseudo likelihood function $L_{p}$ (more precisely, the pseudo log-likelihood function) for person $p$ is defined as

$$
\log L_{p}=\sum_{i=1}^{I}\left[w_{p i} \log P_{p i}+\left(1-w_{p i}\right) \log \left(1-P_{p i}\right)\right]
$$

Where $P_{p i}$ is the probability of correct answer of item $i$ for person $p$, and $w_{p i}$ is the score of person $p$ on item $i$. In the case of non-missing item responses, the scores $w_{p i}$ is either equal to 1 (for a correct answer) or 0 (for an incorrect answer). Lord (1974) argues that in the case of missing item responses for multiple-choice items with $M$ alternatives, the scores $w_{p i}$ could be set to $1 / M$, and the resulting pseudo likelihood function (3) is optimized. As argued above, by ignoring missing item responses, the values of the missing responses would be scored by $w_{p i}=P_{p i}\left(\theta_{p, M}\right)$, where $\theta_{p, M}$ is the ability estimate for a person $p$ based on the non-missing item responses. If the missing item is treated as incorrect, a score $w_{p i}=0$ would be chosen. These two cases should be regarded as two extreme cases of treating missing item treatment responses. Interim assumptions can be expressed by a sensitivity parameter $\rho=0$ (scoring missings responses as incorrect) and $\rho=1$ (treating missing responses as ignorable) that can be examined in the context of a sensitivity analysis. In this approach, we define the score 
$w_{p i}=\rho P_{p i}\left(\theta_{p, M}\right)$, given a preliminary ability estimate $\theta_{p, M}$. Then, ability estimates are derived as a function of the parameter $\rho$. Note that one can also use a marginal pseudo likelihood function in (3) in which a distribution for ability values $\theta$ is assumed. This technique of sensitivity analysis is frequently used for imputations of nonignorable missing data (Resseguier et al. 2011; van Buuren 2012). It should be noted that the parameter $\rho$ itself is not estimable but must be fixed for the estimation. This is also supported by the fact that the maximization of the pseudo likelihood function with respect to $\rho$ can only result in $\rho=0$ or $\rho=1$.

The likelihood function (3) can be written as

$$
L_{p}=\prod_{i=1}^{I} L_{p i}=\prod_{i=1}^{I}\left\{P_{p i}^{w_{p i}}\left(1-P_{p i}\right)^{1-w_{p i}}\right\}
$$

Consequently, the likelihood for person $p$ on item $i$ contributes with $L_{p i}=P_{p i}^{w_{p i}}\left(1-P_{p i}\right)^{1-w_{p i}}$. The missing item responses can therefore be regarded as fuzzy data with values 0 and 1 and associated membership function $m_{p i}(0)=1-w_{p i}$ or $m_{p i}(1)=w_{p i}$ (Denoeux 2011b). The multiplicative term $L_{p i}$ is also denoted as partial membership (see Gruhl and Erosheva 2015, p. 21). Alternatively, for fuzzy data, an additive likelihood term according to $L_{p i}=w_{p i} P_{p i}+\left(1-w_{p i}\right)\left(1-P_{p i}\right)$ has been proposed (see Denoeux 2011b), which is also denoted as mixed membership (Gruhl and Erosheva 2015, p. 20).

\subsubsection{Estimation}

For the estimation in the pseudo-likelihood approach, scores $w_{p i}$ are required in Equation (3). The scores are computed for missing item responses based on a first scaling. In this first step, missing item responses are treated as ignorable problems, and individual person ability estimates are obtained (e.g. weighted likelihood estimates, see Warm 1989), given the observed data and predetermined item difficulties. Alternatively, plausible values could be drawn from the individual posterior distribution (Mislevy 1991). The pseudo likelihood approach (3) with fixed weights $w_{p i}$ can be estimated with the usual EM algorithm (see, e.g., Aitkin 2016, von Davier and Sinharay 2014). In this approach, the M-Step remains unchanged compared to evaluations in ordinary IRT models. In the evaluation of the individual likelihood and the expected counts, the pseudo likelihood instead of an ordinary likelihood is used.

\subsection{Modeling the Missing Response Process}

In the second model, missing item responses will be handled similar to the two-dimensional model by Holman and Glas (2005; see also Rose et al. 2010). Let us denote again $\Psi$ as the logistic function, so under the Rasch model (Yen and Fitzpatrick 2006), the probability of a correct item response is given as

$$
P\left(Y_{p i}=1 \mid \theta_{p}\right)=\Psi\left(\theta_{p}-b_{i}\right)
$$

The probability for a missing item response of item $i$ conditional on the response propensity $\xi$ may now also be affected by the unknown item response $Y_{i}$ itself (see Mislevy 2016; Mislevy and Wu 1996; Rosas et al. 2015). Hence, we define

$$
P\left(R_{p i}=1 \mid Y_{p i}=k, \xi_{p}\right)=\Psi\left(\xi_{p}-\beta_{i}-k \delta\right) \quad \text { with } k=0,1
$$

The probability of a non-missing item response (i.e., $R_{p i}=1$ ) depending on the unknown item response $Y_{i}$ can be modeled with $\delta \neq 0$. If $\delta=0$ is chosen, the IRT model for non-ignorable item responses of Holman and Glas (2005) is obtained. It has been shown that the parameter $\rho$ can be identified from data (Guo and Xu 2019; Robitzsch and Lüdtke 2017; see also Sportisse et al. 2020 for a similar model). Also, scaling results can be studied in a sensitivity analysis as a function of a sequence of $\delta$ values. For very small values of $\delta$ (e.g., $\delta=-10)$, one obtains $P\left(R_{p i}=1 \mid Y_{p i}=1, \xi_{p}\right)=1$ and subsequently $P\left(R_{p i}=0 \mid Y_{p i}=1, \xi_{p}\right)=0$. This means that all students who would know the item would provide a 
correct non-missing item response with a probability of 1 . Using the Bayes theorem, it follows that $P\left(Y_{p i}=1 \mid R_{p i}=0 ; \theta_{p}, \xi_{p}\right)=0$. Hence, all missing item responses will be is evaluated as incorrect for a very small value of $\delta$. As in the pseudo-likelihood approach, one can use the proposed model to investigate the extremes of ignoring missing item responses $(\delta=0)$ and scoring missing item responses as incorrect $(\delta=-10)$.

In the proposed item response model, item difficulties $b_{i}$, item parameter $\beta_{i}$ for response tendency as well as the bivariate distribution of $(\theta, \xi)$ are being estimated. In the calculation of the likelihood for item $i$, the probabilities of three disjunctive events $P\left(R_{p i}=1, Y_{p i}=0 \mid \theta_{p}, \xi_{p}\right), P\left(R_{p i}=1, Y_{p i}=1 \mid \theta_{p}, \xi_{p}\right)$ and $P\left(R_{p i}=0 \mid \theta_{p}, \xi_{p}\right)$ are used. The probability of a missing item responses $P\left(R_{p i}=0 \mid \theta_{p}, \xi_{p}\right)$ can be computed as the total probability

$$
P\left(R_{p i}=0 \mid \theta_{p}, \xi_{p}\right)=P\left(R_{p i}=0 \mid Y_{p i}=0 ; \xi_{p}\right) P\left(Y_{p i}=0 \mid \theta_{p}\right)+P\left(R_{p i}=0 \mid Y_{p i}=1 ; \xi_{p}\right) P\left(Y_{p i}=1 \mid \theta_{p}\right)
$$

which can be calculated based on the item parameters. Hence, missing item responses $Y_{p i}$ are integrated out analogous to missing items in large-scale assessments studies by employing the marginal maximum likelihood estimation method integrated (von Davier and Sinharay 2014; see also Mislevy and Wu 1996; Hanson 2000).

\subsubsection{Estimation}

The proposed IRT model is based on the evaluation of item response probabilities conditional on the latent variables $\theta$ and $\xi$. For observed correct item responses, the probability is given by (using Equations (5) and (6))

$$
P\left(Y_{p i}=1, R_{p i}=1 \mid \theta_{p}, \xi_{p}\right)=P\left(R_{p i}=1 \mid Y_{p i}=1, \xi_{p}\right) \cdot P\left(Y_{p i}=1 \mid \theta_{p}\right)=\Psi\left(\xi_{p}-\beta_{i}-\delta\right) \cdot \Psi\left(\theta_{p}-b_{i}\right)
$$

For observed incorrect item responses, one obtains

$$
P\left(Y_{p i}=0, R_{p i}=1 \mid \theta_{p}, \xi_{p}\right)=P\left(R_{p i}=1 \mid Y_{p i}=0, \xi_{p}\right) \cdot P\left(Y_{p i}=0 \mid \theta_{p}\right)=\Psi\left(\xi_{p}-\beta_{i}\right) \cdot \Psi\left(-\theta_{p}+b_{i}\right)
$$

For missing item responses, one obtains using Equation (7)

$$
P\left(R_{p i}=0 \mid \theta_{p}, \xi_{p}\right)=\Psi\left(-\xi_{p}+\beta_{i}\right) \cdot \Psi\left(-\theta_{p}+b_{i}\right)+\Psi\left(-\xi_{p}+\beta_{i}+\delta\right) \cdot \Psi\left(\theta_{p}-b_{i}\right)
$$

The two-dimensional IRT model can be estimated using an EM algorithm. A two-dimensional distribution for $(\theta, \xi)$ is estimated.

\section{Country Comparison of 4 Countries in PIRLS 2011}

In the following section, a comparison of reading literacy performance in PIRLS 2011 will be conducted using a test booklet for four selected countries Austria (AUT), Germany (GER), France (FRA), and the Netherlands (NLD). The dependence of the country means is investigated depending on different treatments of the missing item responses.

\subsection{Data}

In the following analysis, item responses of booklet 13 in PIRLS 2011 (i.e., the "PIRLS Reader") consisting of 35 items ( 15 multiple-choice items with 4 answer alternatives; 20 constructed response items) were used. For this booklet, item responses of 968 Austrian, 809 German, 901 French, and 802 Dutch students were available. For simplicity, all polytomous items were dichotomized, where only the highest scores were recoded as correct.

Descriptive analyses show that the average proportion of missing item responses varied considerably between items and countries (AUT: 11.2\%, DEU: 7.9\%, FRA: 13.6\%, NLD: 2.7\%). Missing proportions for constructed response items were much higher than for multiple-choice items (e.g., for 
AUT: constructed response: $17.7 \%$, multiple-choice: $2.6 \%$ ). When scoring missing item responses as incorrect, Austrian students provided on average $55.1 \%$ correct item responses and slightly surpassed France $(53.7 \%)$ but reached significantly lower results than German students (63.0\%) and Dutch students $(64.4 \%)$.

\subsection{Analysis}

For the country comparison, six different IRT models (referred to as models M1, .., M6) were specified. Student weights were taken into account in marginal maximum likelihood estimation. For a simplified description of the results, country means in each IRT model were transformed so that Austrian students had a country mean of 500 and a country standard deviation of 100 . The trait distributions for Germany, France, and the Netherlands were subjected to the same transformation, so that country comparisons in all IRT models should always be interpreted relative to the performance of Austria.

In model M1 (that corresponds to the official procedure in PIRLS), a unidimensional Rasch model with four groups (i.e., the four countries AUT, GER, FRA, and NLD) was specified in which missing item responses were scored as incorrect. In model M2, missing item responses were ignored, which means that they were omitted in the computation of the marginal likelihood. As an extension to model M2, model M3 was a two-dimensional Rasch model with two latent variables of ability $\theta$ and the response tendency $\xi$. In model M4, a pseudo likelihood approach of the Rasch model was specified in which missing item responses for multiple-choice items with four answer alternatives were scored with the score $w_{p i}=0.25$. In contrast, missing item responses for constructed response items were scored as incorrect (i.e., with a score $w_{p i}=0$ ). In models M1 to M4, item parameters and the distribution parameters for the trait(s) in the four countries are concurrently estimated.

In models M5 and M6, in a first analysis, missing item responses were scored as incorrect, and common item parameters for all four countries were obtained, which were subsequently fixed in a second step. In model M5, the pseudo likelihood approach was used for the Rasch model, in which missing item responses were scored as $w_{p i}=\rho P_{p i}\left(\theta_{p, M}\right)$, where $\theta_{p, M}$ is the person ability that was computed based on the non-missing item responses. The person ability $\theta_{p, M}$ was a random draw from the individual posterior distribution. The country means were calculated depending on the sensitivity parameter $\rho=0,0.01, \ldots, 0.99,1$. In model M6, the two-dimensional model M3 is extended by modeling the missingness process dependence of the absence on the item response itself. This model was calculated depending on the sensitivity parameter $\delta=-10,-9.5, \ldots,-0.5,0$.

For all item response models, the country means of Austria, Germany, France, and the Netherlands were compared. The entire data preparation and estimation of the models was conducted in the software R (R Core Team 2014). For estimating models M1, M2, and M3, the R package TAM (Kiefer et al. 2014) was used. The models M4, M5, and M6 were estimated with the rasch.mml2 function of the R package sirt (Robitzsch 2014).

\subsection{Results}

In Table 1, the country means for all models M1 to M6 are shown. It is noteworthy that the two extreme treatments of missing item responses in model M1 (missing item responses were scored as incorrect scored) and model M2 (missing item responses were ignored) resulted in slight differences for Germany and France in comparison to the country mean of Austria (GER: M1: 537.5, M2: 534.2; FRA: M1: 488.7, M2: 492.4), while the different model specification led to significant differences for the Netherlands (NLD: M1: 540.3, M2: 523.4). These findings can be explained by the fact that the proportion of missing item responses for Dutch students $(2.7 \%)$ was substantially lower than for Austrian students (11.2\%). 
Table 1. Country means for Austria (AUT), Germany (GER), France (FRA), and the Netherlands (NLD) in PIRLS 2011 for different treatments of missing item responses.

\begin{tabular}{lcccc}
\hline Model & AUT & GER & FRA & NLD \\
\hline M1: missing = incorrect & 500 & 537.5 & 488.7 & 540.3 \\
M2: missing = ignorable & 500 & 534.2 & 492.4 & 523.4 \\
M3: 2-dim. model & 500 & 534.9 & 492.5 & 524.8 \\
$\begin{array}{l}\text { M4: pseudo likelihood } \\
\text { (for multiple-choice items) }\end{array}$ & 500 & 537.6 & 489.4 & 539.5 \\
M5: pseudo-likelihood & & & & \\
$\quad \rho=0$ & 500 & 537.3 & 488.9 & 539.9 \\
$\rho=0.3$ & 500 & 537.0 & 490.1 & 535.9 \\
$\rho=0.7$ & 500 & 535.9 & 491.8 & 529.5 \\
$\rho=1$ & 500 & 534.6 & 493.1 & 524.0 \\
$\quad$ & & & & \\
M6: 2-dim. model & 500 & 538.0 & 489.1 & 540.7 \\
$\delta=-10$ & 500 & 535.9 & 490.6 & 532.4 \\
$\delta=-1.5$ & 500 & 535.1 & 491.5 & 528.0 \\
$\delta=-0.5$ & 500 & 534.6 & 492.1 & 525.7 \\
$\delta=0$ & & & & \\
\hline
\end{tabular}

Note. $N$ = sample size; FI = linking based on full invariance; $p=$ power used in robust Haebara linking. The mean for Austria was fixed at 500 in all item response models.

In the models M5 and M6, the sensitivity parameters $\rho$ and $\delta$ are varied within the framework of sensitivity analysis that refers to different assumptions of the missing process for item responses. In practice, the findings from Table 1 form the range of the country means between the extremes of treating missing responses as incorrect (model M1) and treating them as ignorable (model M2). In Figure 1, the country means are shown as a function of the sensitivity parameters in models M5 and M6. The ranks of Germany and the Netherlands switch depending on the parameters $\rho$ and $\delta$. Notably, the country means are continuous and monotone functions of the sensitivity parameters $\rho$ and $\delta$, respectively.
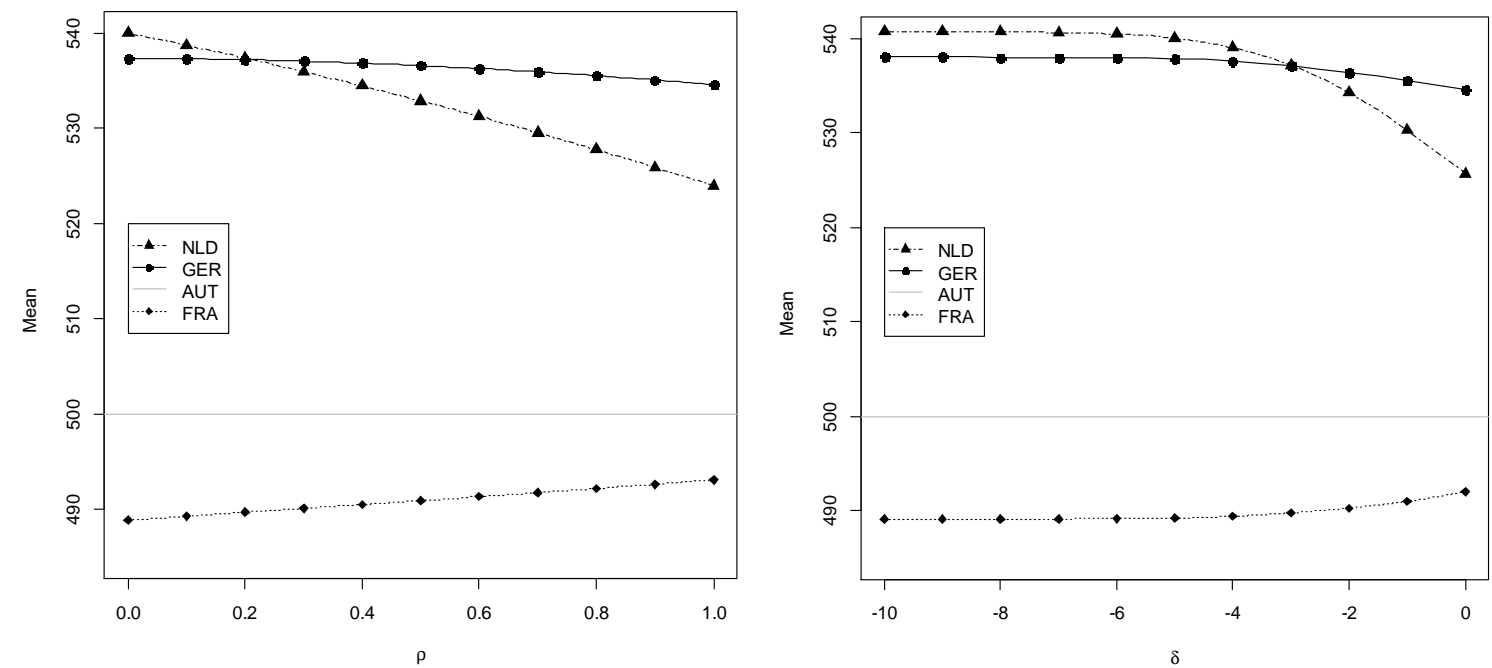

Figure 1. Sensitivity analysis for the country means of Austria (AUT), Germany (GER), France (FRA), and the Netherlands (NLD). Left figure: Pseudo likelihood estimation (model M5) as a function of the sensitivity parameter $\rho$. Right figure: Two-dimensional model M6 as a function of the sensitivity parameter $\delta$. 


\section{Discussion}

In contrast to statements in the literature (Pohl and Carstensen 2013, Pohl et al. 2014), our analyses in PIRLS 2011 showed that ignoring missing item responses has an impact on the central results of a study. Country means varied considerably in different treatments of missing item responses, which raises the question of the choice of a valid scaling approach.

We argued that the criticism against the scoring of missing item responses as incorrect based on test-theoretical arguments (Rose 2013, Pohl et al. 2014) utilizes an intraindividual interpretation of item responses probabilities. In this reasoning, the concepts of aleatoric uncertainty (the statistical model) and epistemic uncertainty (concerning the assessment of data) are erroneously confounded. It was also argued that simulation studies for choosing the "right handling" of missing item responses are irrelevant for choosing a scaling method (see also Rohwer 2013). Since defining an adequate scoring rule for the missing is not empirically determinable, two different IRT models were proposed that served as a sensitivity analysis to different assumptions about missing item responses.

The first alternative one-dimensional IRT model (model M5) is based on a pseudo likelihood approach in which the missing item responses could take values between 0 and 1 . This approach has, however, the disadvantage that for item responses has to be computed. In the second model (model M6), the additional latent response tendency is introduced that is allowed to be correlated with ability. The missingness of an item response is allowed to be dependent on the unknown item response itself.

Typically, the sensitivity analyses performed in this paper are preferred to more complex model specifications, such as pattern mixture models (Rose et al. 2017). When imputing missing data, a range of analysis results can be obtained by varying plausible assumptions on the missingness (van Buuren 2012). If a prior distribution for a sensitivity parameter characterizing the assumptions of the missingness process is specified, then a multi-model inference analysis can be conducted that weighs different theoretical assumptions about the missingness process (Siddique et al. 2012).

Within the scope of our analysis, we have restricted ourselves to the Rasch model. However, the analysis can be extended to more complex IRT models like the 2PL, the 3PL, or the generalized partial credit model. Instead of using a common sensitivity parameter for all items, this parameter can be separately estimated for each individual item or for individual item groups (such as multiple-choice items and constructed response items; see Robitzsch and Lüdtke 2017). If a country-specific missingness process is estimated (i.e., by assuming country-specific $\delta$ parameters), the comparability of the country means obtained from such IRT models could be strongly questioned.

The consequences of treating missing item responses as ignorable or incorrect can also be significant for scaling in longitudinal studies if missing proportions vary by grade, school track, or time points. In IRT models that assume ignorable missing item responses, the meaning of the identified ability remains unclear. If an ability in a particular domain is just defined by the correctly solved items in a test, in our view, a non-circular and more valid definition of ability is obtained. Consequently, it can be concluded that missing item responses cannot be ignorable or partially ignorable.

\section{References}

Aitkin, M.. 2016. Expectation maximization algorithm and extensions. In W. J. van der Linden (Ed.), Handbook of item response theory, Vol. 2: Statistical tools, pp. 217-236. Boca Raton: CRC Press. doi:10.1201/b19166-12.

Bacci, S. and Bartolucci, F.. 2015. A multidimensional finite mixture structural equation model for nonignorable missing responses to test items. Structural Equation Modeling 22(3), 352-365. doi:10.1080/10705511.2014.937376.

Bacci, S., Bartolucci, F., Grilli, L., and Rampichini, C.. 2017. Evaluation of student performance through a multidimensional finite mixture IRT model. Multivariate Behavioral Research 52(6), $732-746$. doi:10.1080/00273171.2017.1361803.

Bartolucci, F., Montanari, G. E., and Pandolfi, S.. 2018. Latent ignorability and item selection for nursing home case-mix evaluation. Journal of Classification 35(1), 172-193. doi:10.1007/s00357-017-9227-9. 
Beesley, L. J., Taylor, J. M. G., and Little, R. J. A.. 2019. Sequential imputation for models with latent variables assuming latent ignorability. Australian \& New Zealand Journal of Statistics 61(2), 213-233. doi:10.1111/anzs.12264.

Bertoli-Barsotti, L. and Punzo, A.. 2013. Rasch analysis for binary data with nonignorable nonresponses. Psicologica 34(1), 97-123.

Debeer, D., Janssen, R., and De Boeck, P.. 2017. Modeling skipped and not-reached items using IRTrees. Journal of Educational Measurement 54(3), 333-363. doi:10.1111/jedm.12147.

Denoeux, T.. 2011a. Maximum likelihood estimation from fuzzy data using the EM algorithm. Fuzzy Sets and Systems 183(1), 72-91. doi:10.1016/j.fss.2011.05.022.

Denoeux, T.. 2011b. Maximum likelihood estimation from uncertain data in the belief function framework. IEEE Transactions on Knowledge and Data Engineering 25(1), 119-130. doi:10.1109/TKDE.2011.201.

Fischer, G. H. and Molenaar, I. W.. 1995. Rasch models. Foundations, recent developments, and applications. New York: Springer. doi:10.1007/978-1-4612-4230-7.

Frey, A., Spoden, C., Goldhammer, F., and Wenzel, S. F. C.. 2018. Response time-based treatment of omitted responses in computer-based testing. Behaviormetrika 45(2), 505-526. doi:10.1007/s41237-018-0073-9.

Fu, Z.-H., Tao, J., and Shi, N.-Z.. 2010. Bayesian estimation of the multidimensional graded response model with nonignorable missing data. Journal of Statistical Computation and Simulation 80(11), 1237-1252. doi:10.1080/00949650903029276.

Glas, C. A. W., Pimentel, J. L., and Lamers, S. M. A.. 2015. Nonignorable data in IRT models: Polytomous responses and response propensity models with covariates. Psychological Test and Assessment Modeling 57(4), 523-541.

Graham, J. W.. 2009. Missing data analysis: Making it work in the real world. Annual Review of Psychology 60, 549-576. doi:10.1146/annurev.psych.58.110405.085530.

Gruhl, J. and Erosheva, E. A.. 2015. A tale of two (types of) memberships: Comparing mixed and partial membership with a continuous data example. In E. M. Airoldi, D. Blei, E. A. Erosheva, and S. E. Fienberg (Eds.), Handbook of mixed membership models and their applications, pp. 15-38. Boca Raton: Chapman \& Hall.

Guo, J. and Xu, X.. 2019. An IRT-based model for omitted and not-reached items. arXiv Preprint arXiv:1904.03767.

Hanson, B.. 2000. IRT parameter estimation using the EM algorithm. Technical report. Retrieved from http://www.b-a-h.com/.

Harel, O. and Schafer, J. L.. 2009. Partial and latent ignorability in missing-data problems. Biometrika 96(1), 37-50. doi:10.1093/biomet/asn069.

Holland, P. W.. 1990. On the sampling theory foundations of item response theory models. Psychometrika 55(4), 577-601. doi:10.1007/BF02294609.

Holman, R. and Glas, C. A. W.. 2005. Modelling non-ignorable missing-data mechanisms with item response theory models. British Journal of Mathematical and Statistical Psychology 58(1), 1-17. doi:10.1111/j.2044-8317.2005.tb00312.x.

Jung, H., Schafer, J. L., and Seo, B.. 2011. A latent class selection model for nonignorably missing data. Computational Statistics \& Data Analysis 55(1), 802-812. doi:10.1016/j.csda.2010.07.002.

Kiefer, T., Robitzsch, A., and Wu, M.. 2014. TAM: Test analysis modules. $\mathrm{R}$ package version 1.0-2. https: / /CRAN.R-project.org/package=TAM.

Köhler, C., Pohl, S., and Carstensen, C. H.. 2015a. Investigating mechanisms for missing responses in competence tests. Psychological Test and Assessment Modeling 57(4), 499-522.

Köhler, C., Pohl, S., and Carstensen, C. H.. 2015b. Taking the missing propensity into account when estimating competence scores: Evaluation of item response theory models for nonignorable omissions. Educational Psychological Measurement 75(5), 850-874. doi:10.1177/0013164414561785.

Kreitchmann, R. S., Abad, F. J., and Ponsoda, V.. 2018. A two-dimensional multiple-choice model accounting for omissions. Frontiers in Psychology 9, 2540. doi:10.3389/fpsyg.2018.02540.

Kuha, J., Katsikatsou, M., and Moustaki, I.. 2018. Latent variable modelling with non-ignorable item nonresponse: Multigroup response propensity models for cross-national analysis. Journal of the Royal Statistical Society. Series A: Statistics in Society 181(4), 1169-1192. doi:10.1111/rssa.12350.

Lord, F. M.. 1974. Estimation of latent ability and item parameters when there are omitted responses. Psychometrika 39(2), 247-264. doi:10.1007/BF02291471.

Lu, J. and Wang, C.. 2020. A response time process model for not-reached and omitted items. Journal of Educational Measurement. Advance online publication, doi:10.1111/jedm.12270. 
Mislevy, R. J.. 1991. Randomization-based inference about latent variables from complex samples. Psychometrika 56(2), 177-196. doi:10.1007/BF02294457.

Mislevy, R. J.. 2016. Missing responses in item response modeling. In W. J. van der Linden (Ed.), Handbook of item response theory, Vol. 2: Statistical tools, pp. 171-194. Boca Raton: CRC Press. doi:10.1201/b19166-10.

Mislevy, R. J. and Wu, P.-K.. 1996. Missing responses and IRT ability estimation: Omits, choice, time limits, and adaptive testing (Research Report No. RR-96-30). Educational Testing Service. doi:10.1002/j.2333-8504.1996.tb01708.x.

Molenaar, I. W.. 1995. Some background for item response theory and the Rasch model. In G. H. Fischer and I. W. Molenaar (Eds.), Rasch models. foundations, recent developments, and applications, pp. 3-14. New York: Springer. doi:10.1007/978-1-4612-4230-7_1.

Molenaar, P. C. M.. 2004. A manifesto on psychology as idiographic science: Bringing the person back into scientific psychology, this time forever. Measurement: Interdisciplinary Research and Perspectives 2(4), $201-218$. doi:10.1207/s15366359mea0204_1.

Okumura, T.. 2014. Empirical differences in omission tendency and reading ability in PISA: An application of tree-based item response models. Educational Psychological Measurement 74(4), 611-626. doi:10.1177/0013164413516976.

Pohl, S. and Becker, B.. 2020. Performance of missing data approaches under nonignorable missing data conditions. Methodology 16(2), 147-165. doi:10.5964/meth.2805.

Pohl, S. and Carstensen, C.. 2012. NEPS technical report - Scaling the data of the competence tests (NEPS Working Paper No. 14). Bamberg: Otto-Friedrich-Universität, Nationales Bildungspanel.

Pohl, S. and Carstensen, C. H.. 2013. Scaling of competence tests in the national educational panel study - many questions, some answers, and further challenges. Journal for Educational Research Online 5(2), 189-216.

Pohl, S., Gräfe, L., and Rose, N.. 2014. Dealing with omitted and not-reached items in competence tests: Evaluating approaches accounting for missing responses in item response theory models. Educational Psychological Measurement 74(3), 423-452. doi:10.1177/0013164413504926.

R Core Team. 2014. R: A language and environment for statistical computing. Vienna, Austria. https://www.R-project.org/.

Resseguier, N., Giorgi, R., and Paoletti, X.. 2011. Sensitivity analysis: When data are missing not-at-random. Epidemiology 22(2), 282-283. doi:10.1097/EDE.0b013e318209dec7.

Robitzsch, A.. 2014. sirt: Supplementary item response theory models. R package version 0.44-48. https: / /CRAN.R-project.org/package=sirt.

Robitzsch, A.. 2016. Zu nichtignorierbaren Konsequenzen des (partiellen) Ignorierens fehlender Item Responses im Large-Scale Assessment [On nonignorable consequences of (partial) ignoring of missing item responses in large-scale assessments]. In B. Suchan, C. Wallner-Paschon, and C. Schreiner (Eds.), PIRLS E TIMSS 2011. Die Kompetenzen in Lesen, Mathematik und Naturwissenschaften am Ende der Volksschule. Österreichischer Expertenbericht, pp. 55-64. Graz: Leykam.

Robitzsch, A. and Lüdtke, O.. 2017. An item response model for omitted responses in performance tests. Talk held at IMPS 2017, Zurich, July 2017.

Rohwer, G.. 2013. Making sense of missing answers in competence tests (NEPS Working Paper No. 30). Bamberg: Otto-Friedrich-Universität, Nationales Bildungspanel.

Rosas, G. and Shomer, Y.. 2008. Models of nonresponse in legislative politics. Legislative Studies Quarterly 33(4), 573-601. doi:10.3162/036298008786403088.

Rosas, G., Shomer, Y., and Haptonstahl, S. R.. 2015. No news is news: Nonignorable nonresponse in roll-call data analysis. American Journal of Political Science 59(2), 511-528. doi:10.1111/ajps.12148.

Rose, N.. 2013. Item nonresponses in educational and psychological assessment. Unpublished dissertation. Friedrich-Schiller-Universität Jena. Online at https:/ / tinyurl.com/y5t82gqz.

Rose, N., von Davier, M., and Nagengast, B.. 2015. Commonalities and differences in IRT-based methods for nonignorable item nonresponses. Psychological Test and Assessment Modeling 57(4), 472-498.

Rose, N., von Davier, M., and Nagengast, B.. 2017. Modeling omitted and not-reached items in IRT models. Psychometrika 82(3), 795-819. doi:10.1007/s11336-016-9544-7.

Rose, N., von Davier, M., and Xu, X.. 2010. Modeling nonignorable missing data with item response theory (IRT). (Research Report No. RR-10-11). Educational Testing Service. doi:10.1002/j.2333-8504.2010.tb02218.x. 
Santos, V. L. F., Moura, F. A. S., Andrade, D. F., and Gonçalves, K. C. M.. 2016. Multidimensional and longitudinal item response models for non-ignorable data. Computational Statistics E Data Analysis 103, 91-110. doi:10.1016/j.csda.2016.05.002.

Siddique, J., Harel, O., and Crespi, C. M.. 2012. Addressing missing data mechanism uncertainty using multiple-model multiple imputation: Application to a longitudinal clinical trial. Annals of Applied Statistics 6(4), 1814-1837. doi:10.1214/12-AOAS555.

Sportisse, A., Boyer, C., and Josse, J.. 2020. Imputation and low-rank estimation with missing not at random data. Statistics and Computing 30(6), 1629-1643. doi:10.1007/s11222-020-09963-5.

Ulitzsch, E., von Davier, M., and Pohl, S.. 2020. Using response times for joint modeling of response and omission behavior. Multivariate Behavioral Research 55(3), 425-453. doi:10.1080/00273171.2019.1643699.

van Buuren, S.. 2012. Flexible imputation of missing data. Boca Raton: CRC Press. doi:10.1201/b11826.

von Davier, M. and Sinharay, S.. 2014. Analytics in international large-scale assessments: Item response theory and population models. In L. Rutkowski, M. von Davier, and D. Rutkowski (Eds.), A handbook of international large-scale assessment: Background, technical issues, and methods of data analysis, pp. 155-174. London: Chapman Hall/CRC Press. doi:10.1201/b16061.

Wainer, H.. 2010. Visual revelations: Schrödinger's cat and the conception of probability in item response theory. Chance 23(1), 53-56. doi:10.1080/09332480.2010.10739793.

Warm, T. A.. 1989. Weighted likelihood estimation of ability in item response theory. Psychometrika 54(3), 427-450. doi:10.1007/BF02294627.

Weeks, J. P., von Davier, M., and Yamamoto, K.. 2016. Using response time data to inform the coding of omitted responses. Psychological Test and Assessment Modeling 58, 671-701.

Yen, W. M. and Fitzpatrick, A. R.. 2006. Item response theory. In R. L. Brennan (Ed.), Educational measurement, pp. 111-154. Praeger Publishers.

Zhou, S. and Huggins-Manley, A. C.. 2019. The performance of the semigeneralized partial credit model for handling item-level missingness. Educational Psychological Measurement 80(6), 1196-1215. doi:10.1177/0013164420918392. 